\title{
Chasing an Unpredictable Quarry
}

\section{Researchers explore the effects of random motion in a pursuit problem in which hounds chase a hare.}

\author{
By Michael Schirber
} $\mathrm{n}$ a classic pursuit-theory problem, a pack of hounds chases a hare. A common assumption for the model is that the hare runs in a straight line or in some other prescribed way. Davide Bernardi from the Italian Institute of Technology, Ferrara, and Benjamin Lindner from Humboldt University of Berlin have added a new twist to this game: a randomly moving hare [1]. They explore how randomness in the hare's path and the energy constraints on the hounds affect the time it takes for the hounds to catch the hare. The results could provide guidance in developing nanorobot swimmers that can steer themselves toward a chosen goal.

Pursuit theory is used in applications ranging from the designing of missile defense systems to the programming of search algorithms. Researchers have also employed the pursuit-theory framework to "microscopic chases," in which a cell moves toward higher concentrations of some resource. Previous pursuit models included some noise in the motion, but a fully random target had not been considered until now.

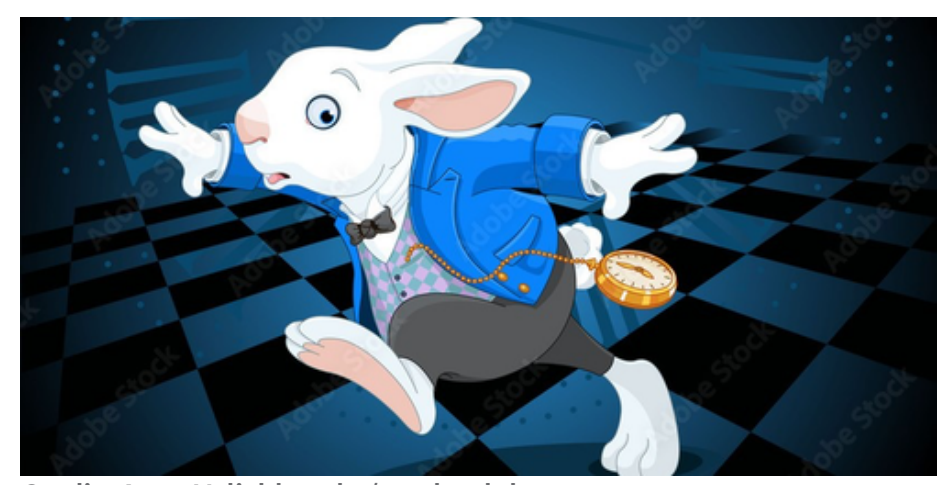

Credit: Anna Velichkovsky/stock.adobe.com
Bernardi and Lindner assume the hare is a Brownian particle that randomly walks over a two-dimensional surface. The hounds, by contrast, are deterministic: they always move in the direction of the hare. Simulations are run until one of the hounds catches up with the hare. Increasing the hare's randomness (or diffusion rate) can delay capture, but only if the randomness is above a critical value determined by the number of hounds. In their analytical modeling, the researchers also included the energy expended by the hounds and found that-for certain consumption rates-a large number of slow-moving hounds can be more efficient than a small pack of fast-moving ones.

Michael Schirber is a Corresponding Editor for Physics based in Lyon, France.

\section{REFERENCES}

1. D. Bernardi and B. Lindner, "Run with the Brownian hare, hunt with the deterministic hounds," Phys. Rev. Lett. 128, 040601 (2022). 